\title{
(C) OPEN ACCESS \\ Management challenges of late presentation Dacron patch infection after carotid endarterectomy
}

\author{
Jie Hua Xu, ${ }_{1}^{1}$ Nishath Altaf, ${ }^{2}$ Patrik Tosenovsky, ${ }^{2}$ Bibombe Patrice Mwipatayi ${ }^{2,3}$
}

'Department of Urology, Royal Perth Hospital, Perth, Western Australia, Australia ${ }^{2}$ Department of Vascular and Endovascular Surgery, Royal Perth Hospital, Perth, Western Australia, Australia

${ }^{3}$ School of Surgery, University of Western Australia, Perth, Western Australia, Australia

Correspondence to

Professor Bibombe

Patrice Mwipatayi

bibombe@iinet.net.au

Accepted 3 October 2017

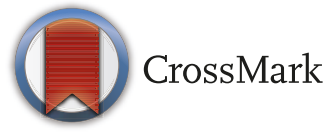

To cite: $\mathrm{Xu}$ JH, Altaf $\mathrm{N}$ Tosenovsky $\mathrm{P}$, et al. BMJ Case Rep Published Online First: [please include Day Month Year]. doi:10.1136/bcr-2017 221541

\section{SUMMARY}

An 83-year-old man presented 4 years after right carotid endarterectomy (CEA) with an infection of his prosthetic Dacron patch. Initial scans (CT angiogram and whole body labelled white cell scan) were clear with no infection or collection noted. Systemically, the patient presented well with no recorded fevers. With an occluded left internal carotid artery and severely stenosed vertebral arteries, surgery presented a high risk of major stroke due to the lack collateral supply and this was discussed extensively. The patient subsequently declined surgical management, and he was monitored closely on an outpatient basis. He presented again a year later with ongoing haemoserous ooze from the CEA site. Subsequently a two-stage procedure was performed, where initially a stent was inserted, followed by patch excision and debridement. A muscle flap was then mobilised over the opening. This new approach to carotid patch infections should gain traction over time as a safer alternative for high-risk patients.

\section{BACKGROUND}

Although carotid prosthetic patch infections are rare, they are associated with high rates of morbidity and mortality when they do occur. Therefore, a high degree of suspicion is needed when patients present with discharge or swelling from an old endarterectomy site. Interestingly, these patients can present systemically well with negative skin swabs, while still harbouring a patch infection. This is the first case to describe a carotid patch infection presenting 4 years after initial endarterectomy, with an atypical organism grown on culture. The definitive treatment involves removing the infected patch and reconstructing the artery, but patient factors will determine whether a redo surgery is appropriate. A two-stage procedure involving stenting, excision of the infected patch and muscle flap mobilisation was used in this patient due to his high risk, and this should be considered a viable alternative in highrisk patients.

\section{CASE PRESENTATION}

An 83-year-old man who underwent a right carotid endarterectomy (CEA) with a Dacron patch in 2011 following an ipsilateral right hemispherical transient ischaemic attack, presented 4 years later in 2015, with bleeding and pus discharging from a sinus tracking down to the wound. His background included a 3-year history of left internal carotid artery (ICA) occlusion with bilateral high-grade vertebral artery stenosis of more than $70 \%$. He had a left hemispherical stroke in 2008 and a longstanding history of hypertension for which he takes two antihypertensive medications.

In September 2015, the patient reported a 12-month history of discharge from his CEA scar, initially managed by his general practitioner with local dressings and oral antibiotics. Examination revealed a sinus at the distal end of his scar that was expressing haemoserous fluid. No underlying fluctuant collection was palpable, but the overlying region was mildly tender with no surrounding erythema. The patient was haemodynamically stable and did not exhibit any fevers or rigours. A CT angiogram (CTA) demonstrated a mural atheroma at the right proximal ICA with no pseudoaneurysm or collection noted. A whole body labelled white cell study with positron emission tomography (PECT)/CT (technetium colloid) was normal with no objective evidence of graft infection related to the previous CEA. Surgical management was offered, but the patient declined. Consequently, the wound was monitored closely in the outpatient clinic, and he was managed on broad-spectrum antibiotics for 12 weeks.

The patient presented again to the vascular department in June 2016 after he noted approximately $500 \mathrm{~mL}$ of haemoserous liquid discharging from the inferior portion of his CEA wound. He was subsequently admitted and investigated with another CTA scan. The CTA demonstrated some irregularity of the proximal right ICA at the region of surgical interest, with mild enhancement of the surrounding soft tissue. But there was no definite evidence of a pseudoaneurysm formation or dissection. The left ICA remained completely occluded with a complete circle of Willis. However, the carotid duplex scan demonstrated a sinus tract extending down to the anterior inferior margin of the Dacron carotid patch (figure 1A, B). There was a focal outpouching in the contour of the inferior aspect of the lumen covered by the patch. A Tc-99m labelled leucocyte scan supplemented with localised planar single photon emission computed tomography (SPECT)-CT of the neck demonstrated active infection in the soft tissue of the sinus track at the right upper neck with involvement of the anterior aspect of the right CEA patch just superior to the hyoid bone (figure $2 \mathrm{~A}, \mathrm{~B}$ ).

\section{TREATMENT}

With an occluded left ICA and high-grade bilateral vertebral stenosis, in our opinion, the patient 


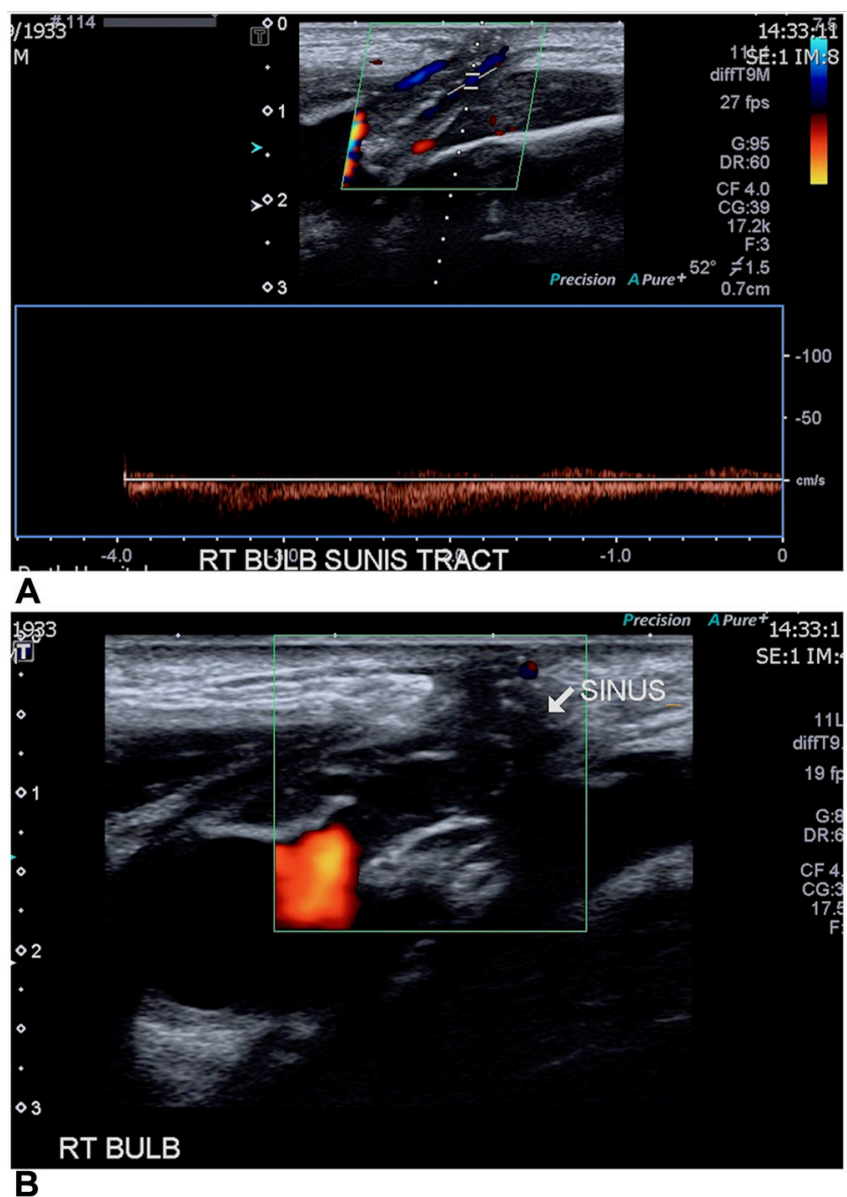

Figure $1 \quad(A, B)$ Carotid duplex scan confirmed a sinus tract extending down to the anterior inferior margin of the Dacron carotid patch.

had a high risk of perioperative stroke if surgical intervention was attempted. However, after yet another episode of bleeding from the wound, surgical treatment was deemed necessary. In the interim, although wound swabs returned with normal skin flora, he was treated with intravenous antibiotics. The patient underwent a two-stage procedure where first, the right ICA was stented with two overlapping covered stents. A covered stent is composed of a metal stent covered by graft material, this surrounding graft material provides a direct barrier against tissue ingrowth and inhibits re-stenosis of the vessel. Using a right femoral approach, the right ICA was cannulated, an outpouching area of the lower part of the patch was clearly visualised and revealed the area of bleeding (figure 3A). Two self-expanding covered stents, the Fluency Plus Endovascular Stent Graft $10 \times 30 \mathrm{~mm}$ (BARD Peripheral Vascular, Tempe, Arizona, USA) and the GORE VIABAHN Endoprosthesis with Heparin Bioactive Surface $10 \times 25 \mathrm{~mm}$ (W L Gore \& Associates,Flagstaff, Arizona, USA), were deployed over a Hi-torque Supra Core guide wire with microglide coating, $0.035,300 \mathrm{~cm}$ length (Abbott Vascular, Santa Clara, California, USA) in the ICA placed beyond the site of the sinus (figure 3B). The arterial puncture site was sealed with a 7 Fr Cordis EXOSEAL Vascular Closure Device (Cordis, Johnson \& Johnson Company, Miami Lakes, Florida, USA).

The patient then underwent an open surgical exposure of the carotid artery using a standard anterior cervical approach under general anaesthesia. The common carotid artery, external carotid artery and ICA were exposed and controlled. The sinus

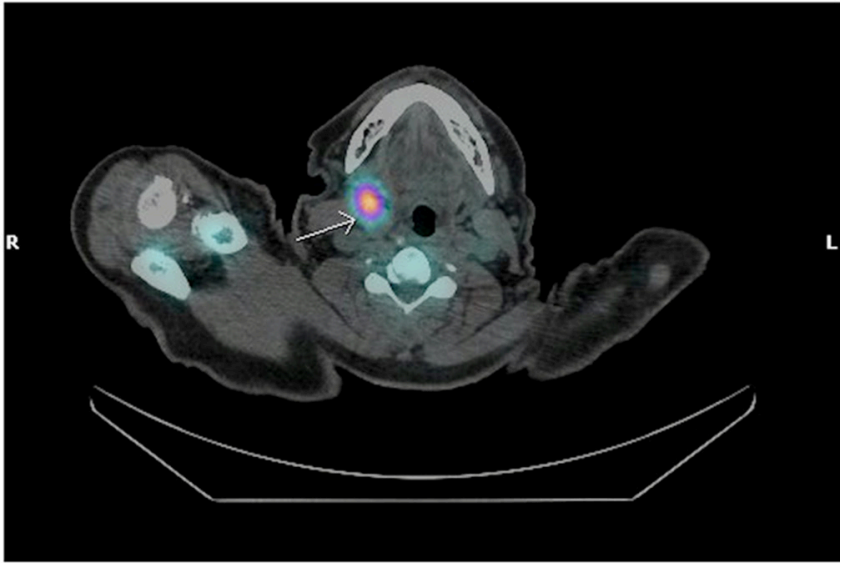

A

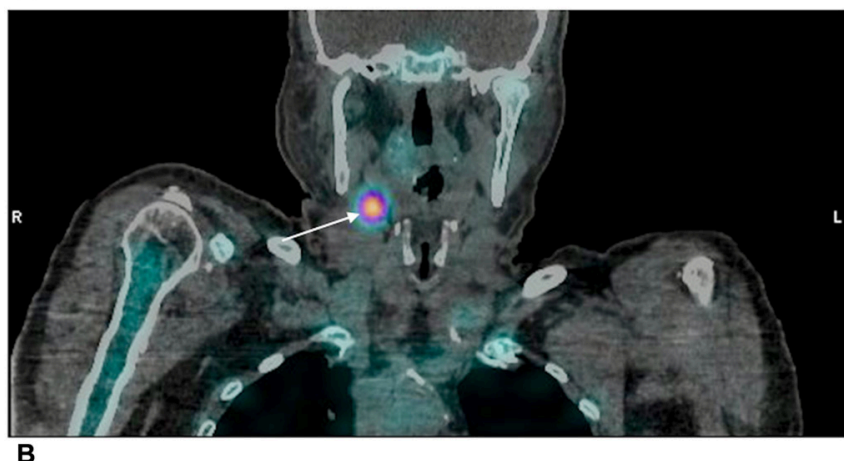

Figure 2 Tc-99m labelled leucocyte scan showed active infection in the soft tissue, with involvement of the anterior aspect of the right carotid endarterectomy (CEA) patch in both coronal (A) and axial (B) views.

was then followed down to the proximal aspect of the carotid patch (figure 4A, B). This was removed and the immediate carotid artery was debrided to the healthy wall. Intraoperatively, a sample of the immediate carotid artery wall was sent for urgent Gram stain; no white cells or bacteria were found to indicate infection. The underlying covered stents were visualised in the healthy carotid artery. After a washout with $0.9 \%$ saline solution, the sternocleidomastoid muscle was mobilised and secured over the exposed covered stent-graft (figure 4C). The platysma and the skin were then closed over a drain (figure 4D).

\section{OUTCOME AND FOLLOW-UP}

Postoperatively, the patient was monitored in the Intensive Care Unit (ICU) and continued on lifelong dual antiplatelet therapy. The excised graft tissue grew Klebsiella pneumoniae and the patient commenced 2 weeks of intravenous piperacillin and tazobactam and was discharged on lifelong oral antibiotics (combination of amoxicillin and clavulanic acid) after consultation with the infectious diseases team. A colour Doppler duplex scan performed the day after the procedure confirmed optimal stent apposition, with no endoleak, outpouching or bleeding point. The patient was discharged home 4 days after the procedure. No major adverse cerebrovascular event occurred at the 30-day follow-up.

\section{DISCUSSION}

Carotid patch infection is a serious complication of CEA, with a risk of morbidity as high as $29 \% .{ }^{12}$ Fortunately, it is a rare occurrence with only 130 cases having been reported in the literature, ${ }^{3}$ 

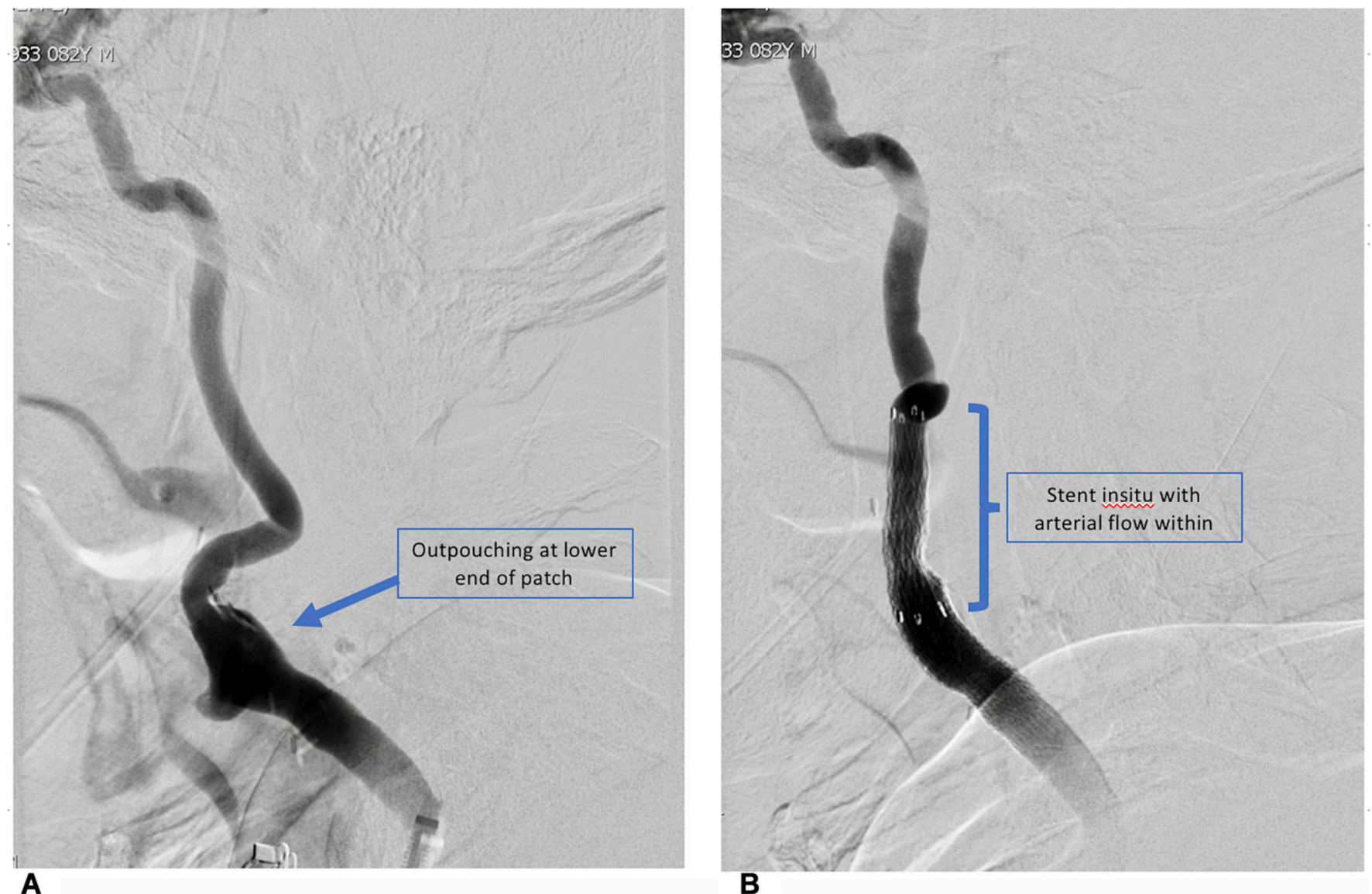

Figure 3 (A) Initial angiography demonstrated an outpouching at the lower end of the patch. (B) Poststent insertion the angiogram was repeated, demonstrating arterial flow through the stent.

affecting roughly $1 \%$ of all synthetic CEA patch repairs. The incidence of Dacron patch infection is quoted to be even lower, at $0.25 \%-0.5 \%{ }^{1}$

While it is possible to repair the carotid artery primarily post-CEA, patch closure is recommended as it markedly reduces re-stenosis rates, perioperative carotid thrombosis and frequency of ipsilateral strokes. ${ }^{4}$ Both vein patch closures and bovine pericardium patches are available, in addition to prosthetic patches. The decision to use a prosthetic Dacron patch is unlikely to have negatively influenced the outcome for this patient. No significant difference in wound infection, stroke, arterial occlusion/rupture or recurrent arterial stenosis has been found between prosthetic and vein patch closures. ${ }^{5}$ Similarly, there are equivalent rates of infection, perioperative bleeding and outpouching formation between bovine pericardial patch closure and Dacron patch use. ${ }^{6}$ Because of the relative ease of sourcing prosthetic patches, vein patch use has declined for initial CEA and is used more frequently when required (ie, infection, redo carotid surgery).

Not surprisingly, the main organisms cultured from carotid patch infections are Gram-positive organisms-predominantly Staph aureus, Staph epidermidis or Pseudomonas. ${ }^{7}$ However, our patient grew K. pneumoniae on the excised Dacron patch, an organism that has not been previously associated with patch infections.

Due to the high risk of mortality associated with redo surgery after CEA, the focus should be on prevention. Reoperation after CEA is associated with higher rates of haemorrhage, stroke and nerve injury that is likely related to the adhesions and scarring obscuring the pre-existing anatomy. ${ }^{8}$ Branches of the vagus nerve and its branches (recurrent and superior laryngeal nerves) are particularly at risk of injury from direct trauma (cauterisation, clamping) or retraction as it lies posterolaterally in the carotid sheath. Injury will manifest itself as vocal hoarseness or loss of an effective cough mechanism. If a high carotid bifurcation is required, the hypoglossal and glossopharyngeal nerve are at risk of injury. Injury to the hypoglossal nerve will cause deviation of the tongue to the ipsilateral side, leading to mastication and speech impediments. Glossopharyngeal nerve injury manifests as dysphagia due to paralysis of the middle pharyngeal constrictor muscles. A branch of the glossopharyngeal nerve (nerve of Hering) innervates the carotid sinus and if extensive carotid bifurcation dissection is performed, can be injured and lead to bradycardia and hypotension intraoperatively. The marginal mandibular branch of the facial nerve runs along the inferior aspect of the ear, and is at risk during high incisions. Injury leads to an ipsilateral smile due to injury to the orbicularis oris muscle.

Possible prophylactic measures to consider include a preoperative course and a longer postoperative antibiotic course in diabetic or immunocompromised patients, adherence to strict sterile surgical techniques, CEA by eversion technique, avoidance of prolonged cervical drains and encouragement of good dental hygiene postoperatively. ${ }^{9}$

While our patient presented with a chronic draining sinus, clinical presentations may be diverse, ranging from patch ruptures and haemorrhage to neck swelling to asymptomatic pseudoaneurysms. The treatment options involve either:

1. Patch preservation with simple debridement. Twenty per cent of reported cases in the literature (26 patients) 


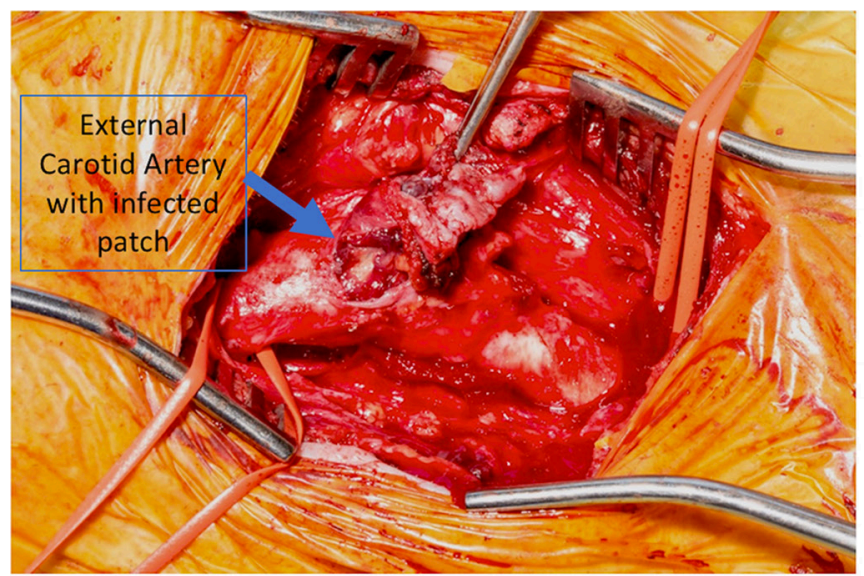

A

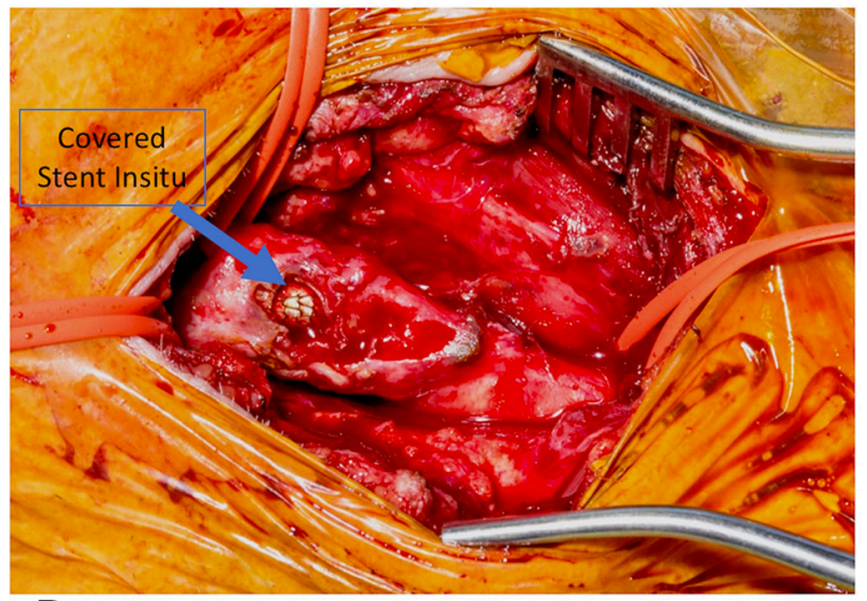

B

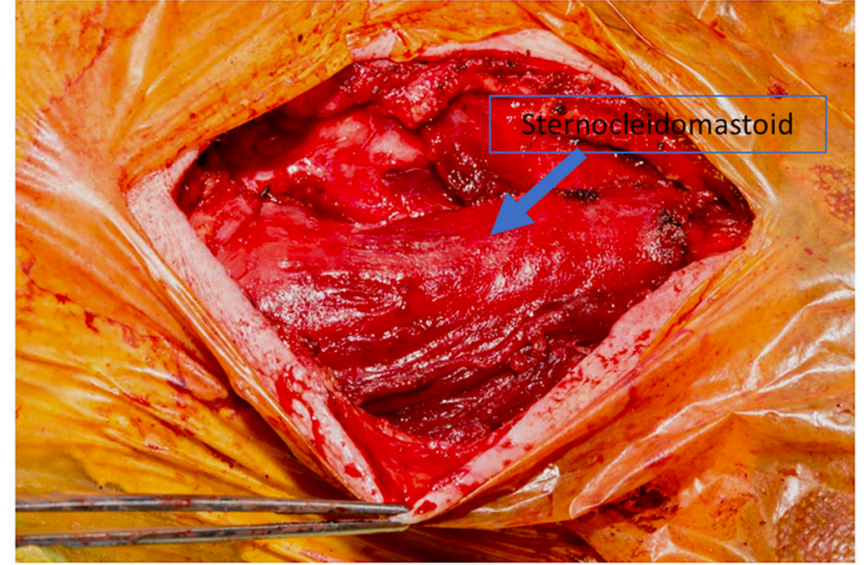

C

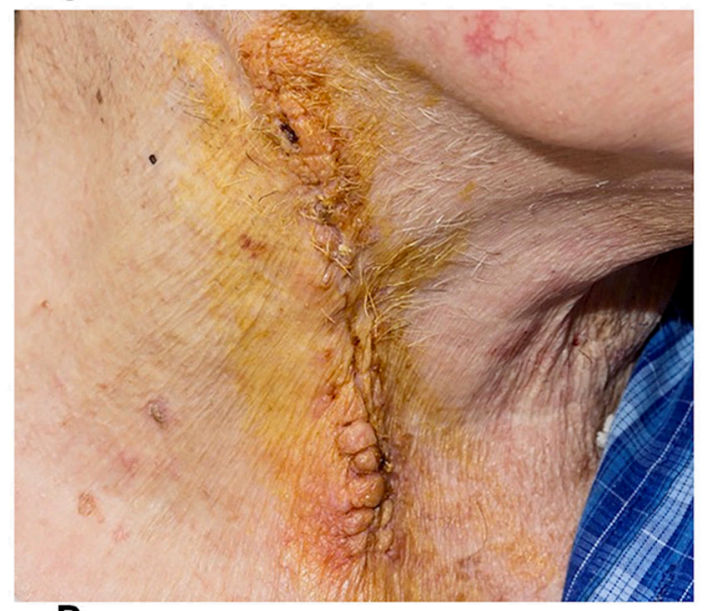

D

Figure 4 (A) Intraoperative exposure of the common carotid, external carotid and internal carotid was performed. (B) The surgical wound was then dissected down to the proximal end of the carotid patch. (C) Subsequently, the sternocleidomastoid muscle was mobilised and secured over the covered stent. (D) The platysma and muscle were closed over the drain.

did not have the infected prosthetic patch excised. A long course of antibiotics was administered instead, and in seven cases, patients had muscle flap coverage. ${ }^{10}$ Three patients were found to suffer a re-infection on follow-up. In $5 \%$ of cases (six patients), no debridement occurred and a covered stent was inserted. Within the short follow-up period of 24 months, no re-infection occurred. Retaining the infected patch is not optimal; therefore, this approach is usually reserved for high-risk patients who are unable to undergo a redo CEA. Interestingly, a case series of 25 CEA graft infections found similar long-term results with patch preservation and patch excision. ${ }^{11}$ However, with a followup period ranging from 1 month to 7 years, it is difficult to ascertain long-term sequelae.

2. Complete patch removal and ligation of the carotid. Only seven cases of carotid ligation and postpatch excision exist in the literature. ${ }^{3}$ One patient suffered a postoperative stroke within 30 days of the procedure, which is consistent with the $40 \%$ stroke risk associated with carotid ligation performed during trauma. Of the seven patients with carotid ligation, none presented again with infection. While not ideal, carotid ligation is a possibility as a last resort, provided the contralateral ICA is patent and the circle of Willis is complete.

3. Complete patch removal and reconstruction of the artery. In $75 \%$ of cases, patients underwent patch excision, the current definitive treatment for prosthetic patch infections. ${ }^{3}$ The subsequent patch site was reconstructed via autologous vein patch, vein bypass, primary closure, native artery bypass, allograft bypass or prosthetic patch/bypass. The subsequent results indicate that prosthetic reconstruction should be avoided. Seven out of the 10 patients returned with a re-infection within 24 months, while one patient suffered a fatal stroke in the perioperative period. Arterial reconstruction via direct closure, venous or arterial graft/bypass is relatively successful with $10 \%$ suffering a stroke or passing away. From the remaining 75 patients, $7 \%$ returned with a late infection.

4. Carotid stenting, followed by patch excision and muscle flap reconstruction. A 2016 flagship paper described a hybrid approach to carotid prosthetic patch infections. ${ }^{12}$ The three-step approach involves inserting a stent, followed by surgical debridement and removal of the infected patch with, or without, a muscle flap. A topical negative pressure dressing then covers the subsequent wound to promote wound granulation and subsequent healing. Six patients were treated successfully, with one requiring a revision to create a sternocleidomastoid flap to achieve full coverage of the wound. At the 5-year follow-up, one patient had an asymptomatic carotid stenosis, but otherwise no negative sequelae were noted.

Perhaps due to the rarity of this complication, no standard management protocol has been set for carotid patch infections and therefore management usually depends on each individual 
surgeon's expertise and judgement. The literature suggests in certain systemically well patients, who are at a high risk for open surgery, a simple debridement with, or without, a stent insertion may be appropriate. Patient selection is easier in hindsight once this strategy has been successful, but it is still difficult to predict the infection course preoperatively. However, if there is an anastomotic haemorrhage, systemic sepsis or outpouching, understandably, surgical intervention must be organised. Prior to any treatment option, if time permits, extensive extracranial and intracranial circulation should be evaluated using CTA and/ or MR angiogram.

Carotid stent infections are rare, with only four cases reported in the literature, ${ }^{13}$ but they are a serious complication when they do occur. Once infected, stents act as a medium for bacteria to attach to the arterial wall, causing arterial wall destruction, thrombosis formation and eventual wall thinning and pseudoaneurysm. This results in both septic embolisation, arterial wall rupture and significant mortality. Management involves antibiotics, and removal of the infected stent is recommended. Little exists in the literature regarding the likelihood of covered stents picking up infection from an infected CEA patch site. In this case, our patient was commenced on lifelong antibiotics and remained infection-free at 1 month follow-up.

After failing intravenous antibiotic therapy, the patient was found to be actively bleeding from the wound site on the ward. Therefore, surgical intervention was required to control the bleeding and control the infection. In this patient with a chronically occluded left ICA and highly stenotic vertebral arteries, clamping the right ICA for patch excision and reconstruction would have been wrought with danger, carrying a high risk of debilitating stroke. Evidently, ligating the ICA was out of the question. Extensive discussions were held in multidisciplinary meetings, with final consensus being the three-stage procedure described above, that negated the risk associated with

\section{Learning points}

- Carotid patch infections are a rare, but serious complication of carotid endarterectomies (CEAs).

- A high degree of suspicion is needed for patients presenting with discharge or swelling from a previous CEA site. These patients can present systemically well with negative skin wound swabs, but this does not exclude a patch infection.

- Dacron infections can present more than 4 years following the initial procedure.

- Treatment is case dependent, but definitive treatment involves removing the infected prosthetic patch with artery reconstruction. Patient factors will determine whether redo surgery is appropriate.

- Prosthetic reconstructions should not be attempted.
ICA clamping. By inserting a stent graft, an intraluminal seal is created, covering the anastomosis and allowing the infected patch to be safely excised without the need for carotid clamping or ligation.

This is the only case in the literature describing a Dacron patch infection occurring over 4 years post-CEA. To our knowledge, it is also the only reported case involving $K$. pneumoniae as the primary infective organism.

Contributors Design and editing of the manuscript. JHX performed the literature review and analysed the current data, drafted and revised the paper. NA performed the literature review and analysed the current data, drafted the management of the patient and revised subsequent versions. PT reviewed and revised the paper. BPM planned and conceived the paper, analysed the current data, drafted and revised the paper. All authors gave final approval before submission.

Competing interests None declared.

Patient consent Obtained.

Provenance and peer review Not commissioned; externally peer reviewed.

Open Access This is an Open Access article distributed in accordance with the Creative Commons Attribution Non Commercial (CC BY-NC 4.0) license, which permits others to distribute, remix, adapt, build upon this work non-commercially, and license their derivative works on different terms, provided the original work is properly cited and the use is non-commercial. See: http://creativecommons.org/ licenses/by-nc/4.0/

(c) BMJ Publishing Group Ltd (unless otherwise stated in the text of the article) 2017. All rights reserved. No commercial use is permitted unless otherwise expressly granted.

\section{REFERENCES}

1 Knight BC, Tait WF. Dacron patch infection following carotid endarterectomy: a systematic review of the literature. Eur J Vasc Endovasc Surg 2009;37:140-8.

2 Asciutto G, Geier B, Marpe B, et al. Dacron patch infection after carotid angioplasty. A report of 6 cases. Eur J Vasc Endovasc Surg 2007;33:55-7.

3 Mann CD, McCarthy M, Nasim A, et al. Management and outcome of prosthetic patch infection after carotid endarterectomy: a single-centre series and systematic review of the literature. Eur J Vasc Endovasc Surg 2012;44:20-6.

4 Rerkasem K, Rothwell PM. Patch angioplasty versus primary closure for carotid endarterectomy. Cochrane Database Syst Rev 2009:CD000160.

5 Rerkasem K, Rothwell PM. Patches of different types for carotid patch angioplasty. Cochrane Database Syst Rev 2010:CD000071.

6 Ho KJ, Nguyen LL, Menard MT. Intermediate-term outcome of carotid endarterectomy with bovine pericardial patch closure compared with Dacron patch and primary closure. J Vasc Surg 2012;55:708.

7 Naylor AR, Payne D, London NJ, et al. Prosthetic patch infection after carotid endarterectomy. Eur J Vasc Endovasc Surg 2002;23:11-16

8 Naughton PA, Garcia-Toca M, Rodriguez HE, et al. Carotid artery reconstruction for infected carotid patches. Eur J Vasc Endovasc Surg 2010;40:492-8.

9 Rizzo A, Hertzer NR, O'hara PJ, et al. Dacron carotid patch infection: a report of eight cases. J Vasc Surg 2000;32:602-6.

10 Naylor R. Management of prosthetic patch infection after CEA. J Cardiovasc Surg 2016:57:137-44

11 Stone PA, Srivastava M, Campbell JE, et al. A 10-year experience of infection following carotid endarterectomy with patch angioplasty. J Vasc Surg 2011;53:1473-7.

12 Thorbjørnsen K, Djavani Gidlund K, Björck M, et al. Editor's choice-long-term outcome after endoVAC hybrid repair of infected vascular reconstructions. Eur J Vasc Endovasc Surg 2016;51:724-32.

13 Son S, Choi NC, Choi DS, et al. Carotid stent infection: a rare but potentially fatal complication of carotid artery stenting. J Neurointerv Surg 2015;7:e14- 
Copyright 2017 BMJ Publishing Group. All rights reserved. For permission to reuse any of this content visit http://group.bmj.com/group/rights-licensing/permissions.

BMJ Case Report Fellows may re-use this article for personal use and teaching without any further permission.

Become a Fellow of BMJ Case Reports today and you can:

- Submit as many cases as you like

- Enjoy fast sympathetic peer review and rapid publication of accepted articles

Access all the published articles

- Re-use any of the published material for personal use and teaching without further permission

For information on Institutional Fellowships contact consortiasales@bmjgroup.com

Visit casereports.bmj.com for more articles like this and to become a Fellow 